\title{
Molecular Origins of Photoinduced Backward Intramolecular Charge Transfer (BICT)
}

Mengyao Hao, ${ }^{\dagger, *}$ Weijie Chi, ${ }^{\ddagger}$ Chao Wang, ${ }^{\ddagger}, \S$, Zhaochao Xu, ${ }^{*, \S}$ Zesheng Li ${ }^{*}{ }^{\dagger}$ and Xiaogang $\operatorname{Liu}^{*,+}$

${ }^{\dagger}$ Key Laboratory of Cluster Science of Ministry of Education, Beijing Key Laboratory of Photoelectronic/Electrophotonic Conversion Materials, School of Chemistry and Chemical Engineering, Beijing Institute of Technology, Beijing 100081, China.

FFluorescence Research Group, Singapore University of Technology and Design, Singapore 487372, Singapore.

${ }^{\S}$ CAS Key Laboratory of Separation Science for Analytical Chemistry, Dalian Institute of Chemical Physics, Chinese Academy of Sciences, 457 Zhongshan Road, Dalian 116023, China 


\section{Contents}

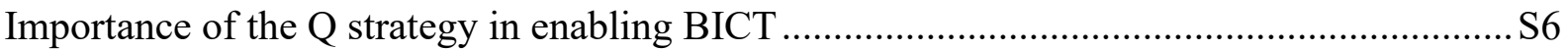

Expanding B, Q, BD and QD series compounds employing different conjugation lengths.... S7

The DDDA strategy employing linear conjugated $\pi$-backbones.......................................S1 1

Negative solvatochromism in BICT compounds ............................................................... S16

Optimized molecular structures, HOMO and LUMO of D1-D9 in ethanol. ........................ S19

Twisted intramolecular charge transfer (TICT) in BICT compounds................................ S20

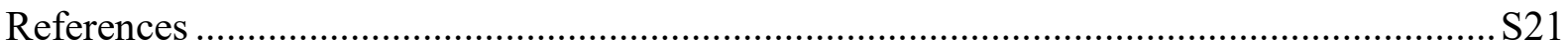




\section{List of Tables}

Table S1. Comparison of selected bond lengths, bond angles, and dihedral angles of DMABN as derived from both XRD experiments ${ }^{1}$ and DFT calculations.

\section{List of Figures}

Figure S1. Molecular structures of derivatives of Brooker's merocyanine (BM). ${ }^{2}$ S6

Figure S2. a) Optimized molecular structures of $\mathbf{B}$ series of compounds. b) Electrostatic static potential (ESP) for the B series; Q denotes the Mulliken charges for the acceptor group. c) Atomic contributions $(\eta)$ to LUMO and HOMO electron densities and the corresponding changes $(\Delta \eta)$ from HOMO to LUMO for the $\mathbf{B}$ series of compounds.

Figure S3. a) Optimized molecular structures of $\mathbf{Q}$ series of compounds. b) Electrostatic static potential (ESP) for the $\mathbf{Q}$ series; Q denotes the Mulliken charges for the acceptor group. c) Atomic contributions $(\eta)$ to LUMO and HOMO electron densities and the corresponding changes $(\Delta \eta)$ from HOMO to LUMO for the $\mathbf{Q}$ series of compounds. S8

Figure S4. a) Optimized molecular structures of BD series of compounds. b) Electrostatic static potential (ESP) for the BD series; Q denotes the Mulliken charges for the acceptor group. c) Atomic contributions $(\eta)$ to LUMO and HOMO electron densities and the corresponding changes $(\Delta \eta)$ from HOMO to LUMO for the BD series of compounds.

Figure S5. a) Optimized molecular structures of QD series of compounds. b) Electrostatic static potential (ESP) for the QD series; Q denotes the Mulliken charges for the acceptor group. c) Atomic contributions $(\eta)$ to LUMO and HOMO electron densities and the corresponding changes $(\Delta \eta)$ from HOMO to LUMO for the QD series of compounds. S10

Figure S6. a) Optimized molecular structures of LA1-LD1 in ethanol. b) Electrostatic static potential (ESP) surfaces of LA1-LD1; Q denotes the Mulliken charge at the acceptor site. c) Atomic contributions $(\eta)$ to LUMO and HOMO electron densities and the corresponding changes $(\Delta \eta)$ from HOMO to LUMO in LA1-LD1; the size of pink/purple circles is proportional to the atomic contribution; only contributions greater than 0.02 are shown. d) Molecular Structures of LA-LD series of compounds. e) $\Delta \eta$ of cyano groups in all compounds. f) $\Delta \eta$ of amino groups in all compounds.

Figure S7. a) Optimized molecular structures of LA2-LD2 in ethanol. b) Electrostatic static potential (ESP) surfaces of LA2-LD2; Q denotes the Mulliken charge at the acceptor site. c) Atomic contributions $(\eta)$ to LUMO and HOMO electron densities and the corresponding changes $(\Delta \eta)$ from HOMO to LUMO in LA2-LD2; the size of pink/purple circles is proportional to the atomic contribution; only contributions greater than 0.02 are shown..... S13 
Figure S8. a) Optimized molecular structures of LA3-LD3 in ethanol. b) Electrostatic static potential (ESP) surfaces of LA3-LD3; Q denotes the Mulliken charge at the acceptor site. c) Atomic contributions $(\eta)$ to LUMO and HOMO electron densities and the corresponding changes $(\Delta \eta)$ from HOMO to LUMO in LA3-LD3; the size of pink/purple circles is proportional to the atomic contribution; only contributions greater than 0.02 are shown..... S14

Figure S9. a) Optimized molecular structures of LE series of compounds. b) Electrostatic static potential (ESP) for the LE series; Q denotes the Mulliken charges for the acceptor group. c) Atomic contributions $(\eta)$ to LUMO and HOMO electron densities and the corresponding changes $(\Delta \eta)$ from HOMO to LUMO for the LE series of compounds.

Figure S10. A comparison of calculated $\left[\lambda_{\text {cal }}(\mathrm{nm})\right.$; red circles $]$ and experimental values $\left[\lambda_{\mathrm{ex}}\right.$ (nm); black squares] for V1-V6 in tetrahydrofuran. S17

Figure S11. a) Optimized molecular structures, LUMO, HOMO of V1-V6 in ethanol. b) Dipole moments in the ground and excited states of V1-V6 in ethanol. c) UV-vis absorption spectra of V1 in tetrahydrofuran (THF), acetonitrile (CAN) and methanol (MeOH), and a comparison of calculated $\left(\lambda_{\text {cal }} ; \mathrm{nm}\right)$ and experimental values $\left(\lambda_{\text {ex }} ; \mathrm{nm}\right)$ of the peak UV-vis absorption wavelengths in different solvents.

Figure S12. Molecular structures, changes of atomic contributions $(\Delta \eta)$ from HOMO to LUMO at both D (or "Part 1" as highlighted in the blue box") and A (or "Part 2" as highlighted in the pink box), optimized molecular structures, HOMOs, LUMOs of D1-D9 in ethanol. The positive/negative $\Delta \eta$ at $\mathrm{D} / \mathrm{A}$, respectively, corroborates the BICT characteristics of these compounds.

Figure S13. (a) The excited-state potential energy surfaces of DADQ along the rotation of the donor and accepter as a function of dihedral angles $\left(\theta_{1}\right.$ and $\left.\theta_{2}\right)$, respectively. (b) The distribution of HOMO (H) and LUMO (L) of the locally-excited (LE) and TICT states for DADQ calculated at the M062X/ss-SMD level of theory in ethanol. The oscillator strength $(f)$ is shown in the inset. S20 
Table S1. Comparison of selected bond lengths, bond angles, and dihedral angles of DMABN as derived from both XRD experiments ${ }^{1}$ and DFT calculations.

\begin{tabular}{|c|c|c|c|}
\hline 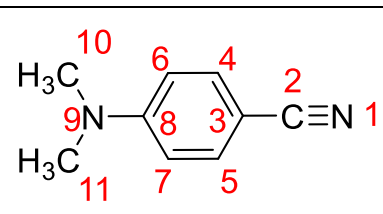 & Parameters & Experiment (XRD) & Calculations (DFT) \\
\hline \multirow[t]{11}{*}{ Bond length $(\AA)$} & $\mathrm{N} 1-\mathrm{C} 2$ & 1.146 & 1.152 \\
\hline & $\mathrm{C} 2-\mathrm{C} 3$ & 1.438 & 1.424 \\
\hline & $\mathrm{C} 3-\mathrm{C} 4$ & 1.392 & 1.399 \\
\hline & $\mathrm{C} 3-\mathrm{C} 5$ & 1.398 & 1.399 \\
\hline & C4-C6 & 1.375 & 1.377 \\
\hline & $\mathrm{C} 5-\mathrm{C} 7$ & 1.380 & 1.377 \\
\hline & C6-C8 & 1.413 & 1.417 \\
\hline & $\mathrm{C} 7-\mathrm{C} 8$ & 1.413 & 1.417 \\
\hline & C8-N9 & 1.368 & 1.360 \\
\hline & N9-C10 & 1.449 & 1.453 \\
\hline & N9-C11 & 1.449 & 1.453 \\
\hline Bond angle $\left({ }^{\circ}\right)$ & $\angle \mathrm{C} 10 \mathrm{~N} 9 \mathrm{C} 11$ & 118 & 120 \\
\hline \multirow[t]{3}{*}{ Dihedral angle $\left(^{\circ}\right)$} & $\angle \mathrm{C} 2 \mathrm{C} 3 \mathrm{C} 4 \mathrm{C} 6$ & 0 & 0 \\
\hline & $\angle \mathrm{C} 6 \mathrm{C} 8 \mathrm{~N} 9 \mathrm{C} 10$ & 7 & 1 \\
\hline & $\angle \mathrm{C} 7 \mathrm{C} 8 \mathrm{~N} 9 \mathrm{C} 11$ & -3 & -1 \\
\hline
\end{tabular}




\section{Importance of the $Q$ strategy in enabling BICT}

The importance of the quinoid moiety in activating BICT and thereby enabling negative solvatochromism is also reflected by the Brooker's merocyanines. ${ }^{2}$ For example, the neutral form of these dyes all possesses quinoid moieties (next to the carbonyl oxygen) in their Lewis structures (Figure S1).

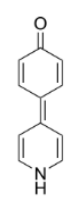

ON-para

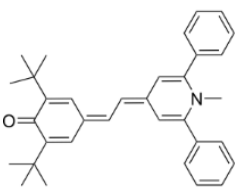

NO-DP

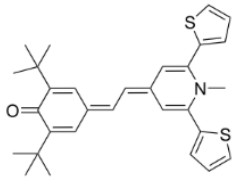

NO-DT

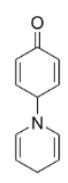

ON-ipso

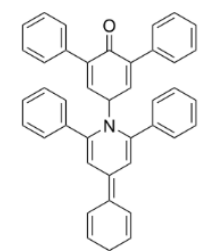

B30

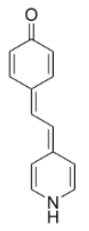

BM

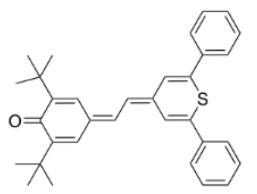

SO-DP

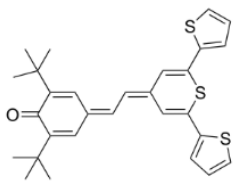

SO-DT
OO-DP
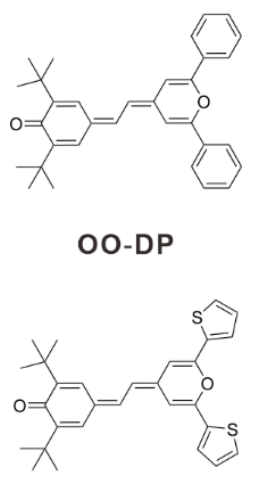

OO-DT

Figure S1. Molecular structures of derivatives of Brooker's merocyanine (BM). ${ }^{2}$ 
Expanding B, Q, BD and QD series compounds employing different conjugation lengths.

a
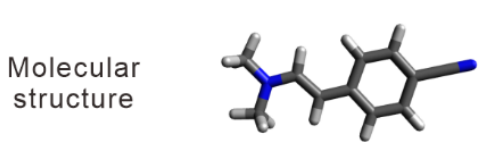

b

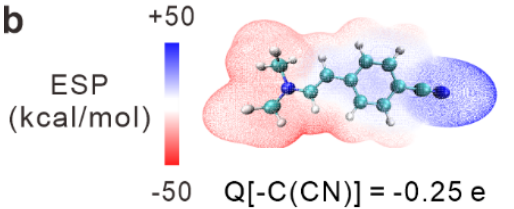

C Atomic contributions

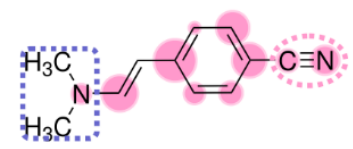

$8.4 \%$

$17.4 \%$
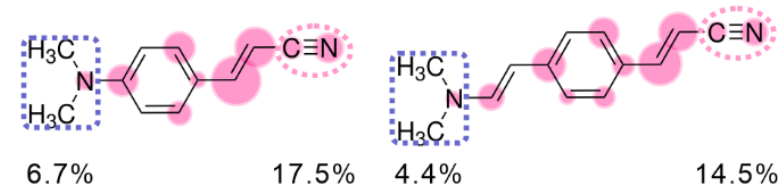

(HOMO)

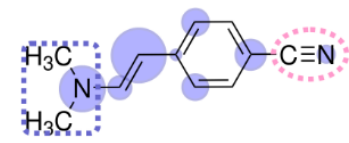

$29.4 \%$

$4.5 \%$

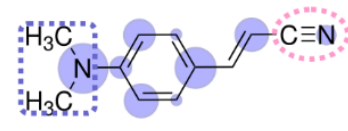

$34.3 \%$

$5.2 \%$

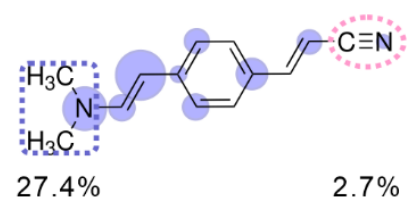

$\Delta \eta \quad-21.0 \%$

$-27.6 \%$

$-22.9 \%$

$11.8 \%$

Figure S2. a) Optimized molecular structures of $\mathbf{B}$ series of compounds. b) Electrostatic static potential (ESP) for the B series; Q denotes the Mulliken charges for the acceptor group. c) Atomic contributions $(\eta)$ to LUMO and HOMO electron densities and the corresponding changes $(\Delta \eta)$ from HOMO to LUMO for the $\mathbf{B}$ series of compounds. 
a
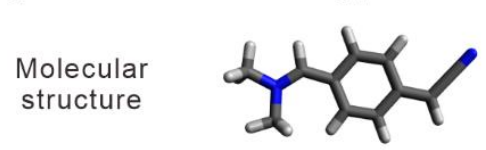

b

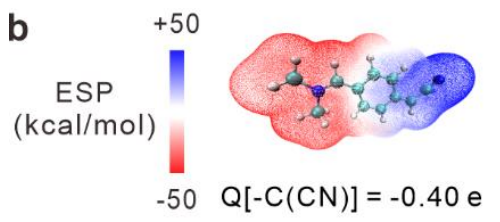

C Atomic contributions $\stackrel{\eta}{(L \cup M O)}$
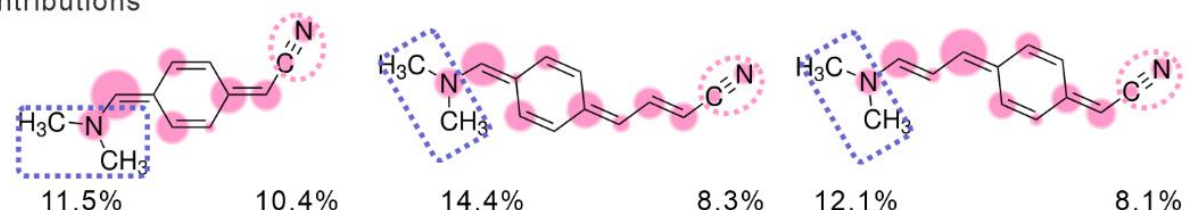

$\stackrel{\eta}{\mathrm{HOMO}}$
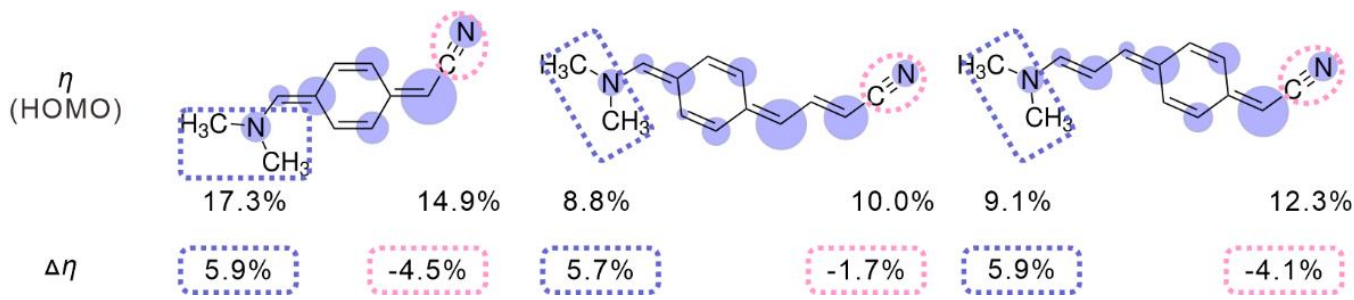

Q2
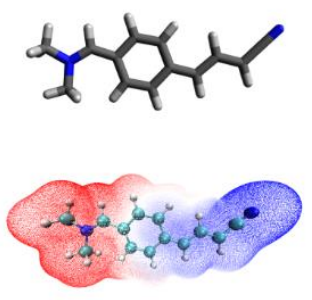

$Q[-C(C N)]=-0.42 e$
Q3
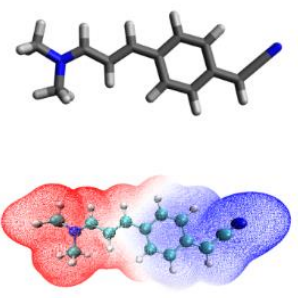

$\mathrm{Q}\left[-\mathrm{C}(\mathrm{CN})_{2}\right]=-0.44 \mathrm{e}$

Figure S3. a) Optimized molecular structures of $\mathbf{Q}$ series of compounds. b) Electrostatic static potential (ESP) for the $\mathbf{Q}$ series; $\mathbf{Q}$ denotes the Mulliken charges for the acceptor group. c) Atomic contributions $(\eta)$ to LUMO and HOMO electron densities and the corresponding changes $(\Delta \eta)$ from HOMO to LUMO for the $\mathbf{Q}$ series of compounds. 
a
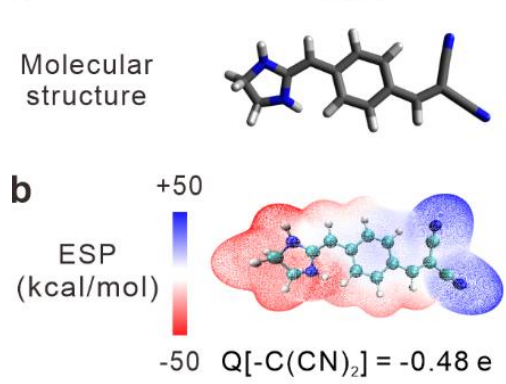

BD2
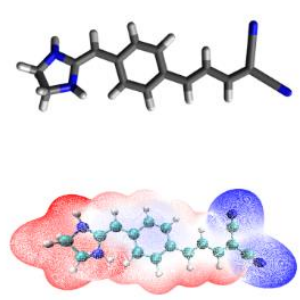

$\mathrm{Q}\left[-\mathrm{C}(\mathrm{CN})_{2}\right]=-0.46 \mathrm{e}$
BD3
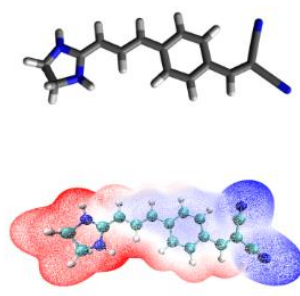

$\mathrm{Q}\left[-\mathrm{C}(\mathrm{CN})_{2}\right]=-0.46 \mathrm{e}$

C Atomic contributions

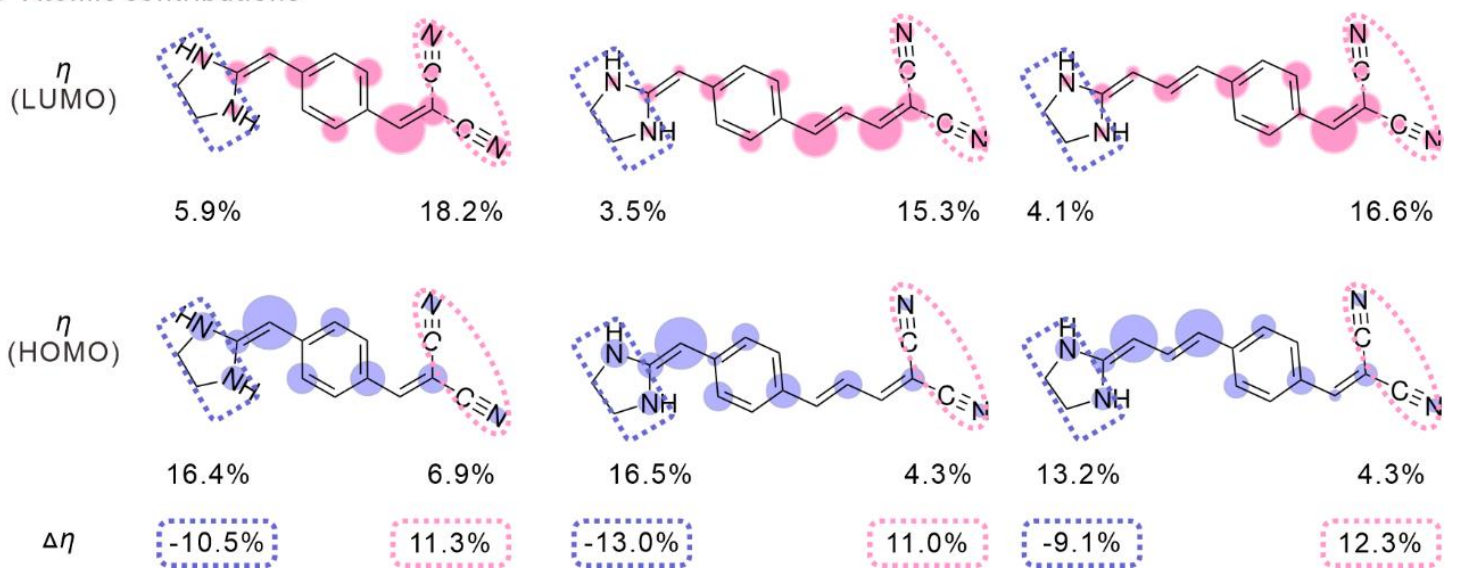

Figure S4. a) Optimized molecular structures of BD series of compounds. b) Electrostatic static potential (ESP) for the BD series; Q denotes the Mulliken charges for the acceptor group. c) Atomic contributions $(\eta)$ to LUMO and HOMO electron densities and the corresponding changes $(\Delta \eta)$ from HOMO to LUMO for the BD series of compounds. 
a
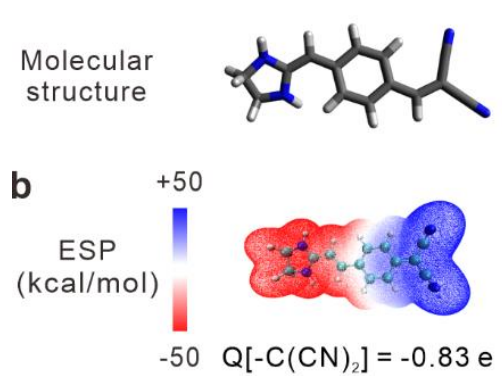

QD2
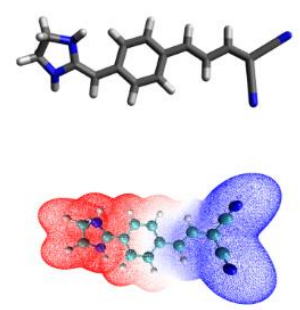

$\mathrm{Q}\left[-\mathrm{C}(\mathrm{CN})_{2}\right]=-0.80 \mathrm{e}$
QD3
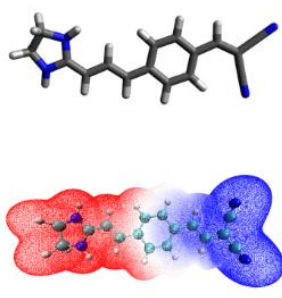

$\mathrm{Q}\left[-\mathrm{C}(\mathrm{CN})_{2}\right]=-0.82 \mathrm{e}$

C Atomic contributions

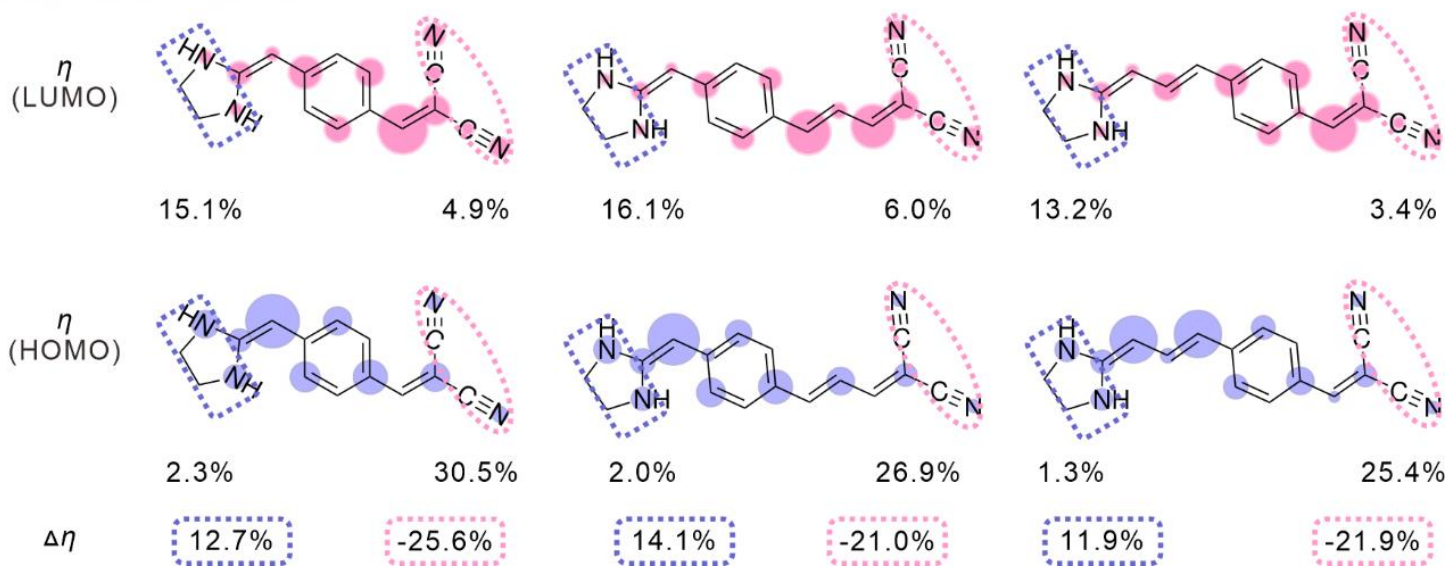

Figure S5. a) Optimized molecular structures of QD series of compounds. b) Electrostatic static potential (ESP) for the QD series; Q denotes the Mulliken charges for the acceptor group. c) Atomic contributions $(\eta)$ to LUMO and HOMO electron densities and the corresponding changes $(\Delta \eta)$ from HOMO to LUMO for the QD series of compounds. 


\section{The DDDA strategy employing linear conjugated $\pi$-backbones.}

To verify the DDDA hypothesis in the absence of the benzenoid and quinoid moieties in the $\pi$-bridge on BICT, we designed four series of compounds (LA-LD series) with varied numbers (one or two) of donors (the amino groups) and acceptors (the cyano groups) connected via linear conjugated backbones. Upon optimizing the representative molecules LA1-LD1 (Figure S6a), ESP analysis shows that the degree of ICT in the ground state gradually increased as the number of donors and acceptors increases. For example, the Mulliken charge at the acceptor moiety increases from -0.16 e in LA1 to -0.69 e in LD1 (Figure S6b).

We also performed the atomic contribution analysis to the frontier molecular orbitals of LA1-LD1, and quantified the change of charge density in terms of the difference between HOMO to LUMO both at the D and A sites $(\Delta \eta)$. In the single-donor-single-acceptor compound LA1, the change of atomic contributions at $\mathrm{D}$ is negative $(\eta=-1.9 \%)$, while that at $\mathrm{A}$ is positive $(\eta=7.1 \%)$. These results show that ICT from $\mathrm{D}$ to $\mathrm{A}$ is further enhanced in the $\mathrm{S}_{1}$ state of LA1, which is a typical characteristic of FICT dyes. Moving from LA1 to LD1, we noted that the changes of atomic contributions at the donor sites become increasingly positive, and that at the acceptor site turns increasingly negative. For example, in the DDDA compound LD1, the atomic contribution at the donor sites increases by $8.3 \%$ upon the vertical excitation, while that at the acceptor site drops by $11.9 \%$. In other words, D withdraws charges and A donates charges during photoexcitation. Indeed, the DDDA strategy endows LD1 with the BICT property. 
LA1

Molecular structure
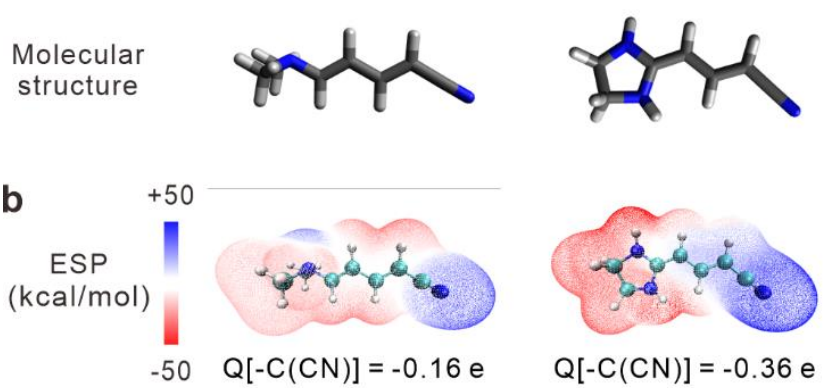

C Atomic contributions $\eta$
$(L \cup M O)$
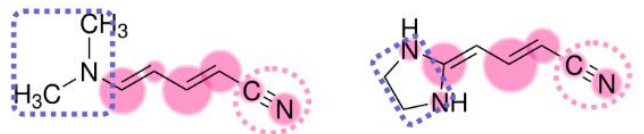

$6.2 \%$

$21.1 \%$

$16.7 \%$
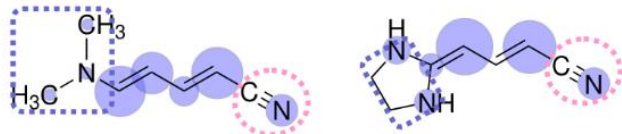

$8.1 \%$

$14.0 \%$

$\Delta \eta$

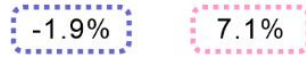

$17.6 \% \quad 14.2 \%$

$-0.9 \% \quad 4.9 \%$
LC1
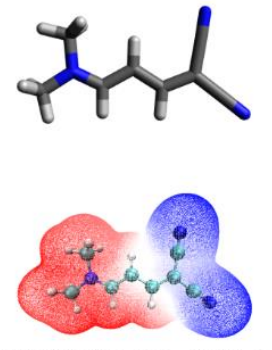

$\mathrm{Q}\left[-\mathrm{C}(\mathrm{CN})_{2}\right]=-0.61 \mathrm{e}$

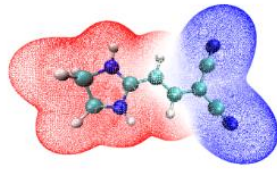

$\mathrm{Q}\left[-\mathrm{C}(\mathrm{CN})_{2}\right]=-0.69 \mathrm{e}$
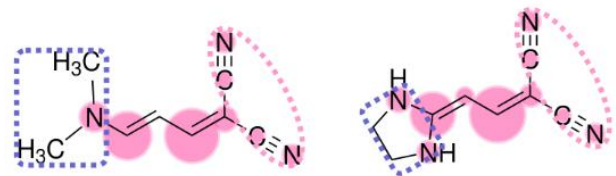

$17.5 \%$

$18.5 \%$

$19.7 \% \quad 17.1 \%$

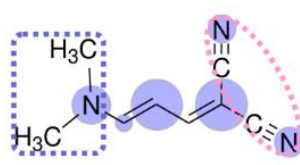

$20.2 \% \quad 24.2 \%$

$-2.7 \%$

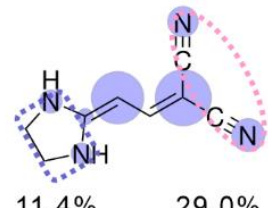

$8.3 \%-11.9 \%$

d
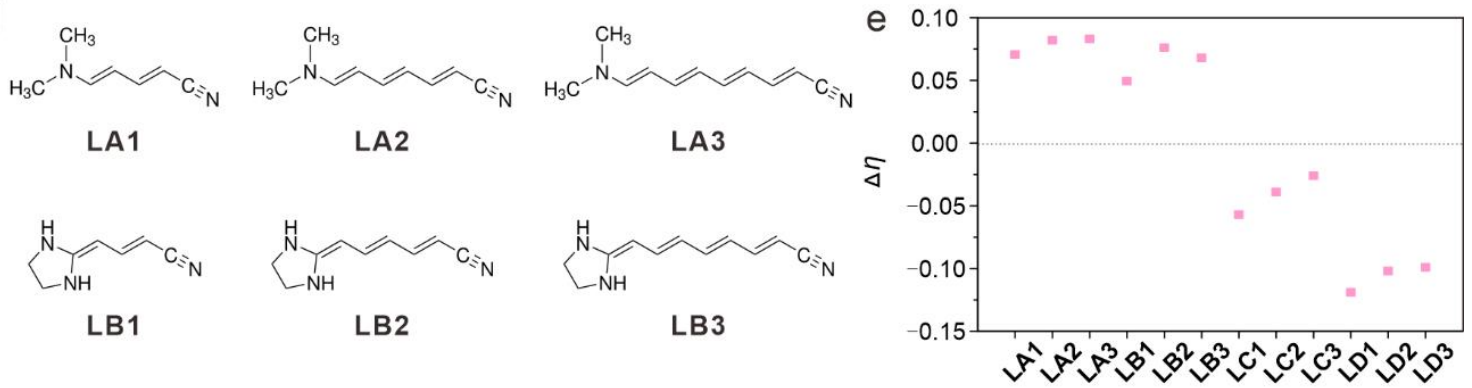

${ }_{H_{3} C^{-N} C_{2}}^{\mathrm{H}_{3} \mathrm{C}}$<smiles>CC(CC1=NCCN1)C(N)N</smiles>

LD1<smiles>C[C@H](N)C(=N)/C=C/C=C1NCCN1</smiles>

LD2<smiles>N#CC(CN)CC=CC=CCC1NCCN1</smiles>

LD3

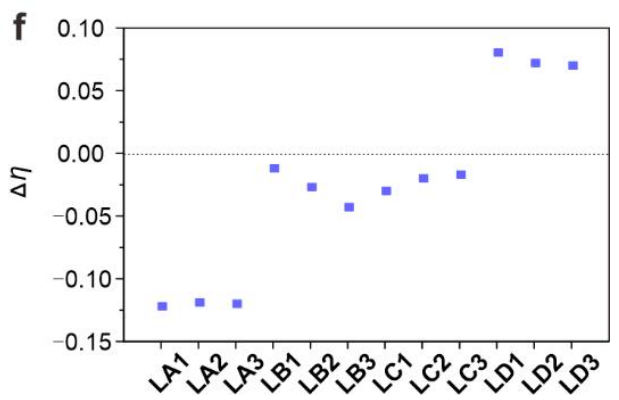

Figure S6. a) Optimized molecular structures of LA1-LD1 in ethanol. b) Electrostatic static potential (ESP) surfaces of LA1-LD1; Q denotes the Mulliken charge at the acceptor site. c) Atomic contributions $(\eta)$ to LUMO and HOMO electron densities and the corresponding changes $(\Delta \eta)$ from HOMO to LUMO in LA1-LD1; the size of pink/purple circles is proportional to the atomic contribution; only contributions greater than 0.02 are shown. d) Molecular Structures of LA-LD series of compounds. e) $\Delta \eta$ of cyano groups in all compounds. f) $\Delta \eta$ of amino groups in all compounds. 
Computational studies of other similar compounds (including LA2-LD2 and LA3-LD3, see Figures S7 and S8) demonstrate the same trend, as shown by the change of the atomic contributions $(\Delta \eta)$ to the frontier molecular orbitals (Figure S6d-f). For example, in all DDDA compounds, $\Delta \eta$ of the cyano moieties (A) is negative, indicating that they are EDG during the photoexcitation (Figure S6e); $\Delta \eta$ of the amino groups (D) fragment is positive, indicating that they withdraw charges (Figure S6f). The role switching of D/A to EWG/EDG during photoexcitation demonstrates that these DDDA compounds are BICT dyes.

a Molecular
structure $\begin{array}{ll}\text { b } & +50 \\ \text { ESP } & \\ \text { (kcal } / \mathrm{mol}) & \\ & \\ & -50\end{array}$
LA2
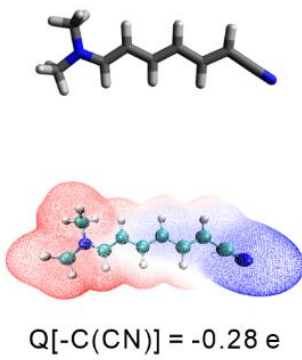
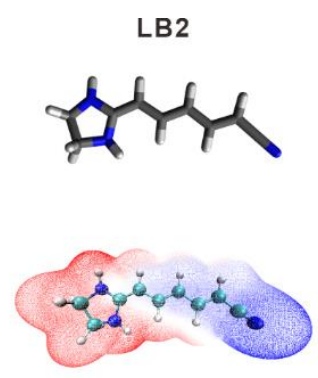

$\mathrm{Q}[-\mathrm{C}(\mathrm{CN})]=-0.32 \mathrm{e}$
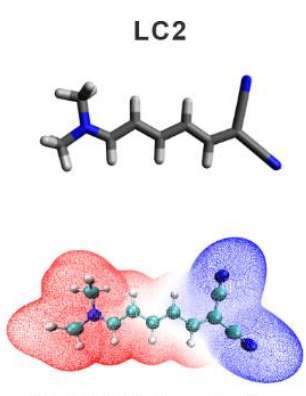

$\mathrm{Q}\left[-\mathrm{C}(\mathrm{CN})_{2}\right]=-0.61 \mathrm{e}$

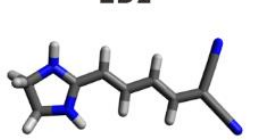

C Atomic contributions

$$
\stackrel{n}{n}(\mathrm{LUM})
$$
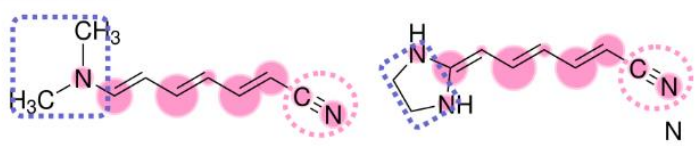

$$
9.7 \%
$$

$15.6 \% \quad 11.6 \%$

$15.0 \%$

$$
\text { (HOMO) }
$$

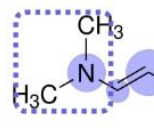

$21.3 \%$

$\Delta \eta$

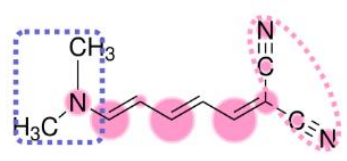

$13.3 \%$

$13.9 \%$

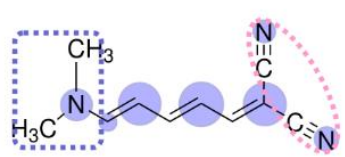

$15.0 \%$

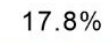

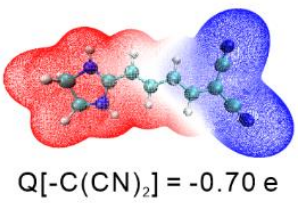

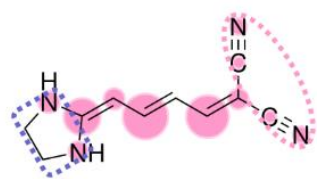

$15.4 \% \quad 12.0 \%$

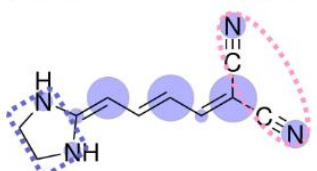

$7.9 \% \quad 22.2 \%$

$7.5 \%$

Figure S7. a) Optimized molecular structures of LA2-LD2 in ethanol. b) Electrostatic static potential (ESP) surfaces of LA2-LD2; Q denotes the Mulliken charge at the acceptor site. c) Atomic contributions $(\eta)$ to LUMO and HOMO electron densities and the corresponding changes $(\Delta \eta)$ from HOMO to LUMO in LA2-LD2; the size of pink/purple circles is proportional to the atomic contribution; only contributions greater than 0.02 are shown. 
a Molecular
structure

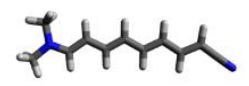

$\begin{array}{ll}\text { ESP } & \\ \text { (kcal/mol) } & +50 \\ & \end{array}$

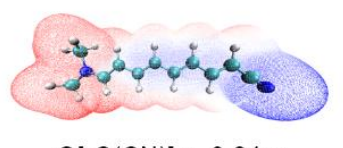

$\mathrm{Q}[-\mathrm{C}(\mathrm{CN})]=-0.24 \mathrm{e}$
LB3
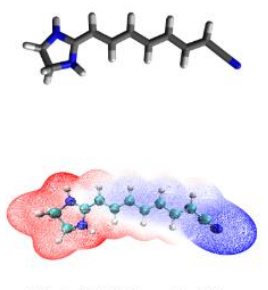

$Q[-C(C N)]=-0.27 e$
LC3
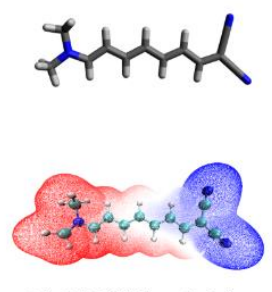

$\mathrm{Q}\left[-\mathrm{C}(\mathrm{CN})_{2}\right]=-0.61 \mathrm{e}$
LD3
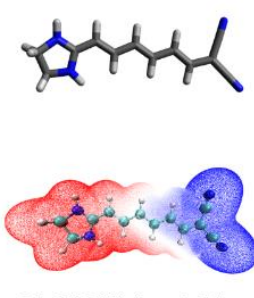

$\mathrm{Q}\left[-\mathrm{C}(\mathrm{CN})_{2}\right]=-0.71 \mathrm{e}$

C Atomic contributions

$\stackrel{n}{(L U M O)}$

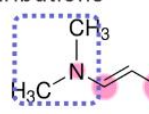

$6.7 \%$

$12.9 \% 8.2 \%$
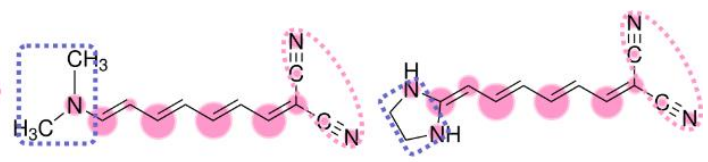

$\stackrel{n}{n}$ (HOMO)
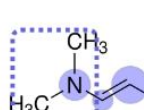

$11.1 \% \quad 12.7 \%$

$8.7 \%$

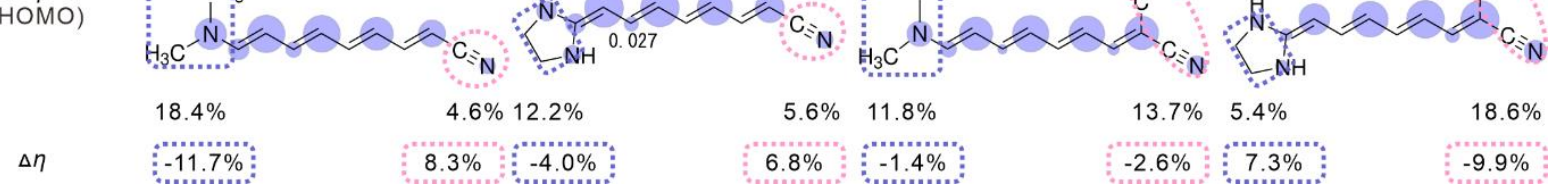

Figure S8. a) Optimized molecular structures of LA3-LD3 in ethanol. b) Electrostatic static potential (ESP) surfaces of LA3-LD3; Q denotes the Mulliken charge at the acceptor site. c) Atomic contributions $(\eta)$ to LUMO and HOMO electron densities and the corresponding changes $(\Delta \eta)$ from HOMO to LUMO in LA3-LD3; the size of pink/purple circles is proportional to the atomic contribution; only contributions greater than 0.02 are shown. 
We also calculated the ICT properties of molecules with triple donors and/or triple acceptors (LE1 and LE2 in Figure S9). However, these compounds did not exhibit the BICT properties, probably due to the non-planarity of the donors and acceptors with respect to the $\pi$-bridge in the resulted compounds.

a
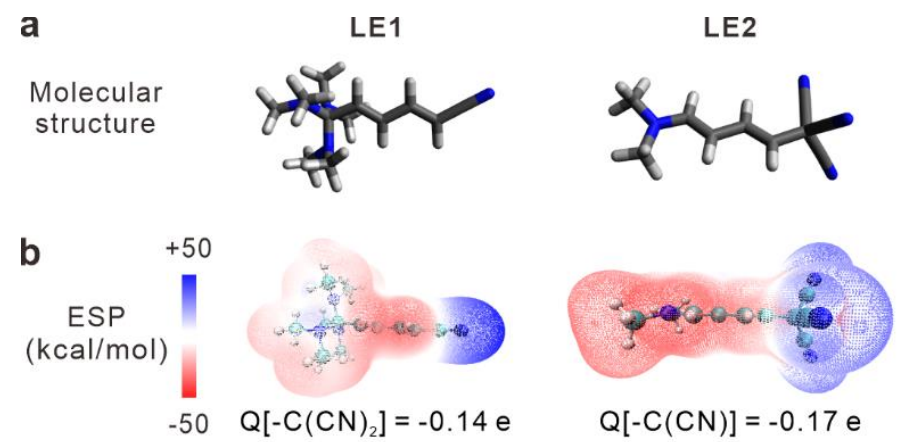

C Atomic contributions

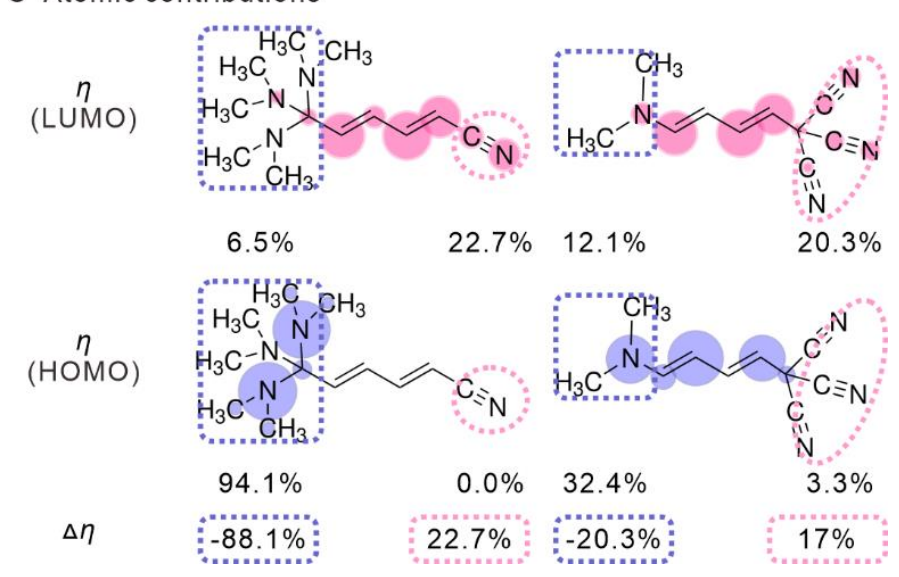

Figure S9. a) Optimized molecular structures of LE series of compounds. b) Electrostatic static potential (ESP) for the LE series; Q denotes the Mulliken charges for the acceptor group. c) Atomic contributions $(\eta)$ to LUMO and HOMO electron densities and the corresponding changes $(\Delta \eta)$ from HOMO to LUMO for the LE series of compounds. 


\section{Negative solvatochromism in BICT compounds}

Our calculations show that a BICT compound possesses a larger dipole moment in the ground state owing to significant charge separation, and a smaller dipole moment in the excited state along with backward ICT. The reduced excited state dipole moment indicates that BICT compounds exhibit negative solvatochromism (blue shifts of UV-vis absorption/emission spectra as solvent polarity increases). This is in contrast to the positive solvatochromism in FICT compounds, which have been widely reported.

To validate our computational predictions as well as explore the unique negative solvatochromism in BICT compounds, we computationally characterized six reported molecules that possess the QDDDA molecular structure, including V1-V6. ${ }^{3}$ Taking THF as an example, the calculated values and experimental values show the same trend and match well (Figure S10). Our calculations show that these compounds present similar BICT nature (Figure S11a). Considerable BICT in these compounds is accompanied by a large decrease in their dipole moments from $\mathrm{S}_{0}$ to $\mathrm{S}_{1}$ states by $\sim 10$ Debye (Figure $\mathrm{S} 11 \mathrm{~b}$ ).

Moreover, TD-DFT calculations in solvents of increasing polarities (including tetrahydrofuran, acetonitrile and methanol) show that V1-V6 possess negative solvatochromism (Figure S11c). For example, calculated peak UV-vis absorption wavelengths $\left(\lambda_{\text {calc }}\right)$ of V1 experience a blue shift from $353 \mathrm{~nm}$ in tetrahydrofuran to $332 \mathrm{~nm}$ in methanol. This blue shift is consistent with experimental observations, thus corroborating the accuracy of our computational analysis and the validity of the QDDDA strategy in enabling BICT. 


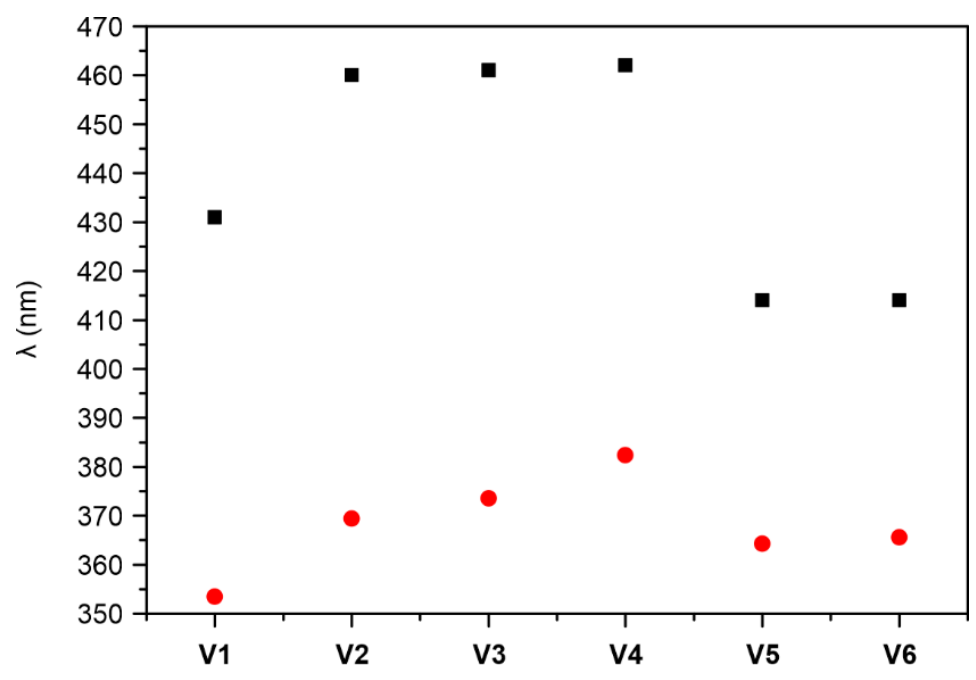

Figure S10. A comparison of calculated $\left[\lambda_{\text {cal }}(\mathrm{nm})\right.$; red circles $]$ and experimental values $\left[\lambda_{\text {ex }}\right.$ (nm); black squares] for V1-V6 in tetrahydrofuran. 

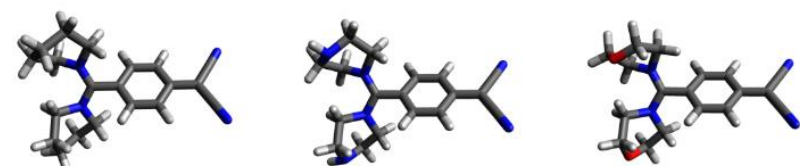

LUMO
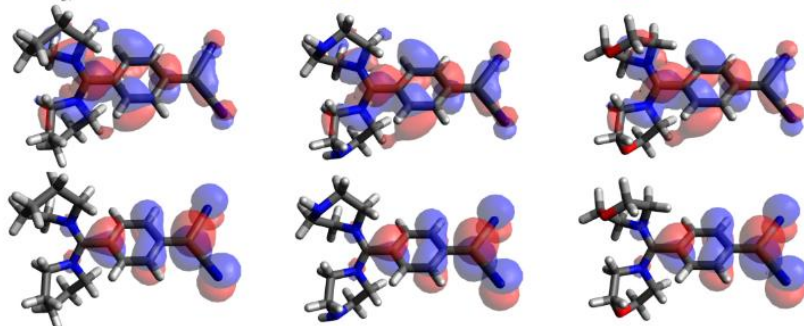

HOMO

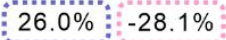

$\Delta \eta$

$25.9 \%-27.9 \%$

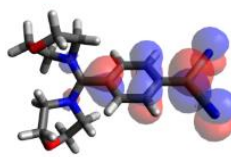

$26.5 \%-27.9 \%$
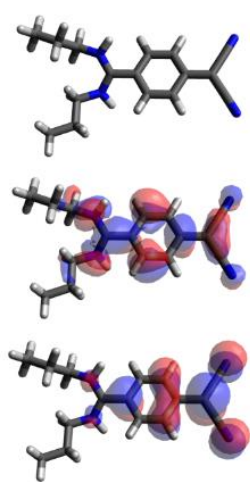

$21.1 \%-27.3 \%$

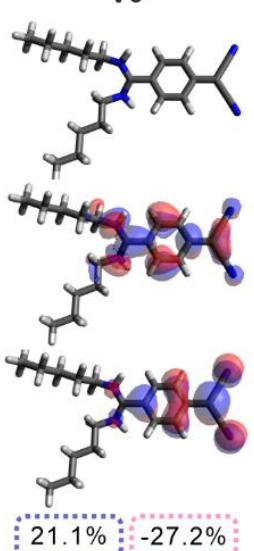

b

$\begin{array}{cc}\underset{\text { Dipole moment }}{\text { Debye) }} & 20.40\left(\mathrm{~S}_{1}\right) \\ & 32.01\left(\mathrm{~S}_{0}\right)\end{array}$

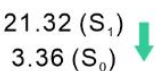

$18.01\left(\mathrm{~S}_{1}\right)$
$29.64\left(\mathrm{~S}_{0}\right)$

$20.61\left(S_{1}\right)$

$31.48\left(\mathrm{~S}_{0}\right)$

$20.66\left(\mathrm{~S}_{1}\right)$ $31.55\left(\mathrm{~S}_{0}\right)$
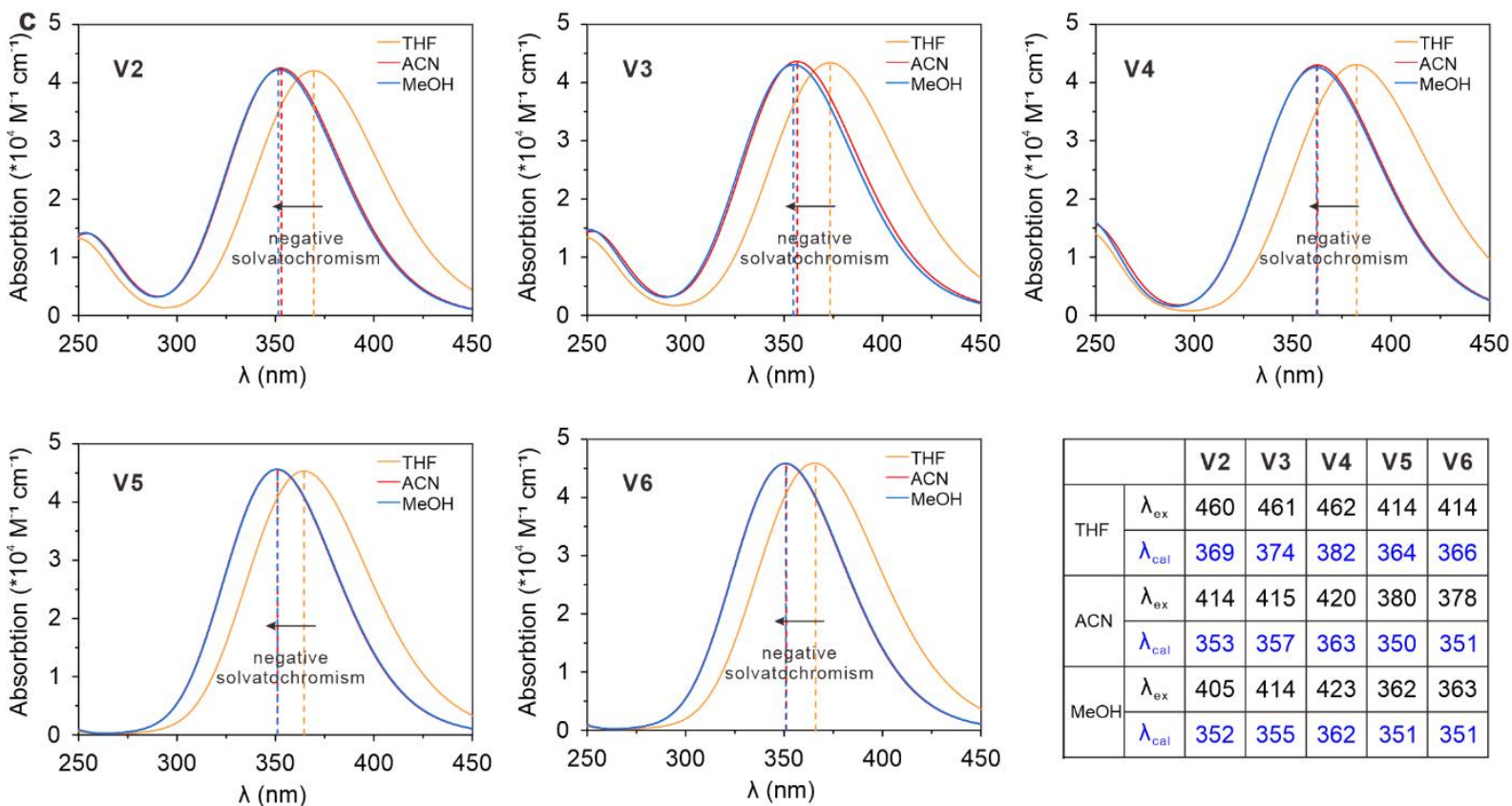

\begin{tabular}{|c|c|c|c|c|c|c|}
\hline \multicolumn{2}{|c|}{} & V2 & V3 & V4 & V5 & V6 \\
\hline \multirow{2}{*}{ THF } & $\lambda_{\text {ex }}$ & 460 & 461 & 462 & 414 & 414 \\
\cline { 2 - 7 } & $\lambda_{\text {cel }}$ & 369 & 374 & 382 & 364 & 366 \\
\hline \multirow{2}{*}{ ACN } & $\lambda_{\text {ex }}$ & 414 & 415 & 420 & 380 & 378 \\
\cline { 2 - 7 } & $\lambda_{\text {cal }}$ & 353 & 357 & 363 & 350 & 351 \\
\hline \multirow{2}{*}{ MeOHy } & $\lambda_{\text {ex }}$ & 405 & 414 & 423 & 362 & 363 \\
\cline { 2 - 7 } & $\lambda_{\text {cal }}$ & 352 & 355 & 362 & 351 & 351 \\
\hline
\end{tabular}

Figure S11. a) Optimized molecular structures, LUMO, HOMO of V1-V6 in ethanol. b) Dipole moments in the ground and excited states of V1-V6 in ethanol. c) UV-vis absorption spectra of V1 in tetrahydrofuran (THF), acetonitrile (CAN) and methanol (MeOH), and a comparison of calculated $\left(\lambda_{\mathrm{cal}} ; \mathrm{nm}\right)$ and experimental values $\left(\lambda_{\mathrm{ex}} ; \mathrm{nm}\right)$ of the peak UV-vis absorption wavelengths in different solvents. 

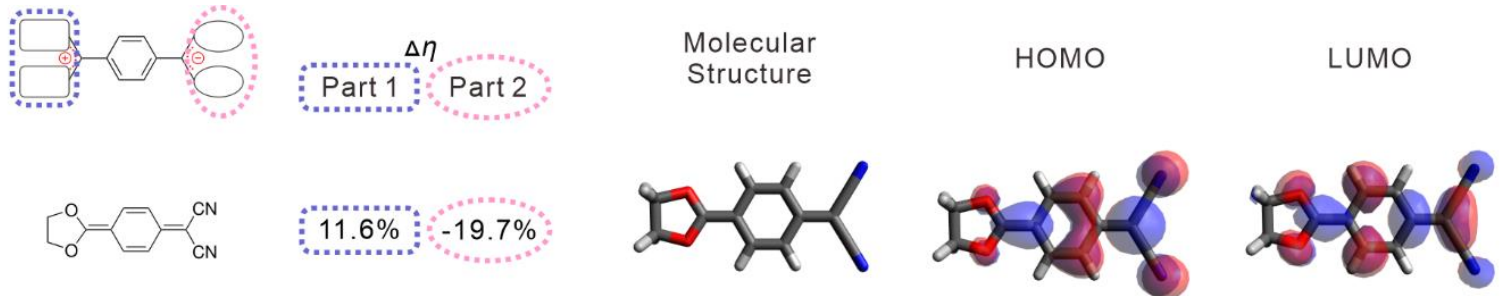

D2

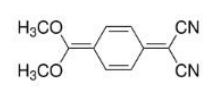

$14.9 \%-22.1 \%$
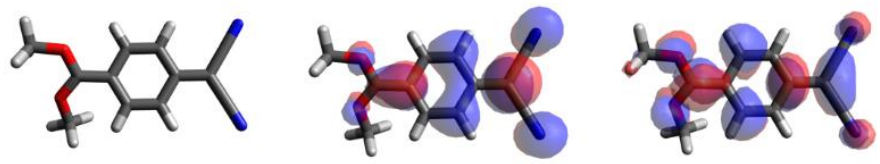

D3

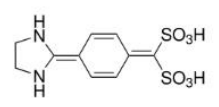

$17.0 \%-18.9 \%$
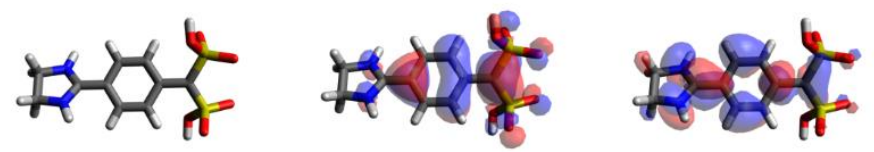

D4<smiles>CC(C)(C)c1ccc(C2=NCCN2)cc1</smiles>
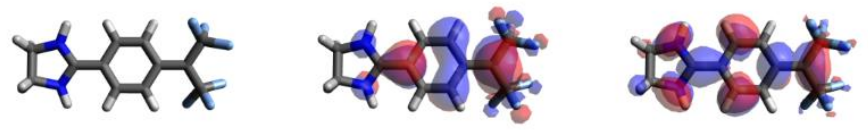

D5<smiles>CC(C#N)C1C=CC(=NC2=NCCN2)N1</smiles>
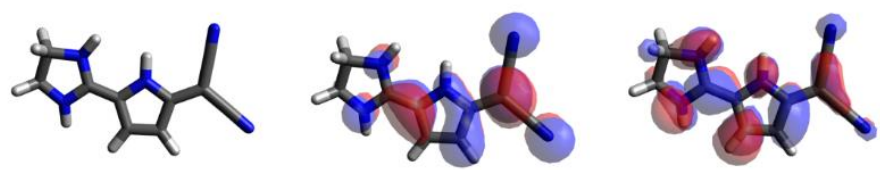

D6

$$
\text { 等 }
$$

$11.4 \%-19.3 \%$
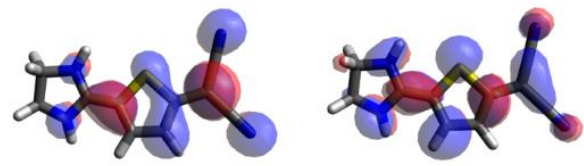

D7

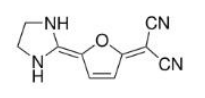

$15.7 \% \quad-21.8 \%$
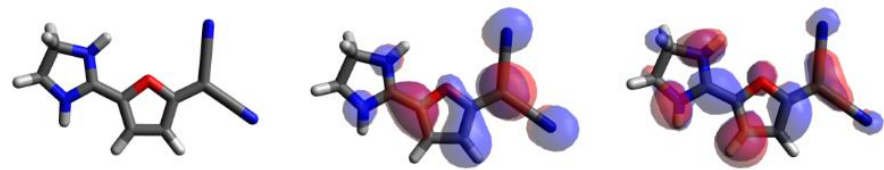

D8

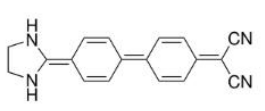

$16.6 \%-31.0 \%$
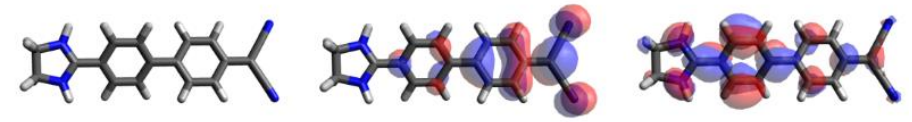

D9<smiles>N#CC(C#N)c1ccc(/C=C/C2C=CC(=C3NCCN3)C=C2)cc1</smiles>
$11.6 \%-26.7 \%$
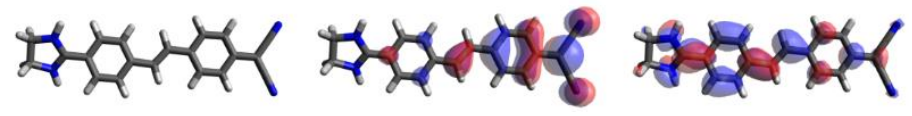

Figure S12. Molecular structures, changes of atomic contributions $(\Delta \eta)$ from HOMO to LUMO at both D (or "Part 1" as highlighted in the blue box") and A (or "Part 2" as highlighted in the pink box), optimized molecular structures, HOMOs, LUMOs of D1-D9 in ethanol. The positive/negative $\Delta \eta$ at $\mathrm{D} / \mathrm{A}$, respectively, corroborates the BICT characteristics of these compounds. 


\section{Twisted intramolecular charge transfer (TICT) in BICT compounds}

To determine whether TICT takes place and which fragment is more likely to rotate in DADQ, the excited-state potential energy surfaces of DADQ along the rotation of the donor and accepter fragments are scanned as a function of the two dihedral angles $\left(\theta_{1}\right.$ and $\left.\theta_{2}\right)$, from $0^{\circ}$ to $90^{\circ}$ with a step size of $10^{\circ}$, respectively. As shown in Figure 12, the rotation of $\theta_{2}$ leads to the formation of the TICT state with a negligible energy barrier. The TICT state is characterized by a complete charge separation from HOMO to LUMO, a zero-oscillator strength $(f)$, and a perpendicular conformation in DADQ.

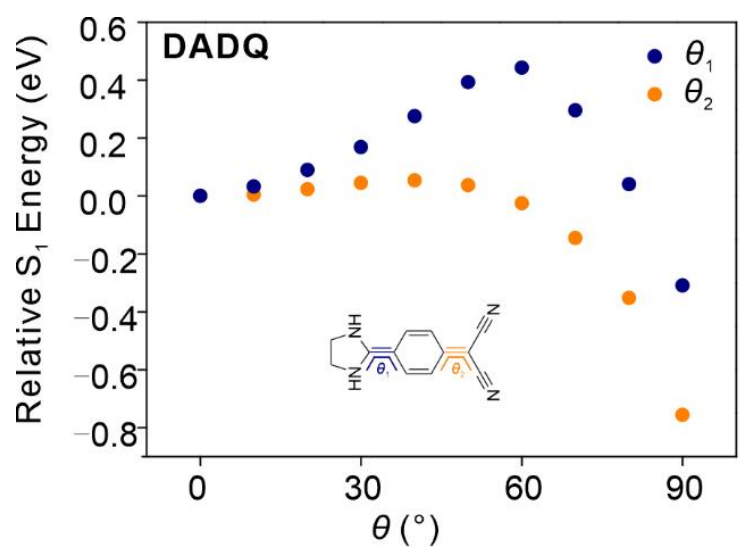

LE state

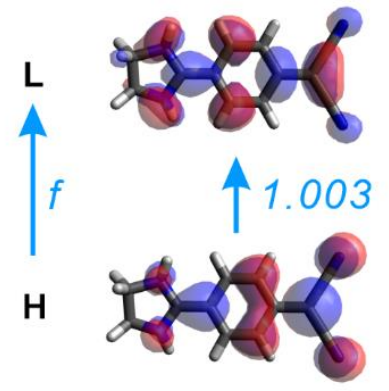

TICT state

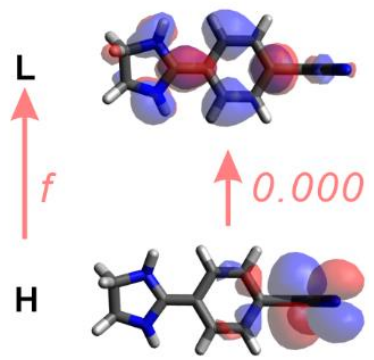

Figure S13. (a) The excited-state potential energy surfaces of DADQ along the rotation of the donor and accepter as a function of dihedral angles $\left(\theta_{1}\right.$ and $\left.\theta_{2}\right)$, respectively. (b) The distribution of HOMO (H) and LUMO (L) of the locally-excited (LE) and TICT states for DADQ calculated at the M062X/ss-SMD level of theory in ethanol. The oscillator strength $(f)$ is shown in the inset. 


\section{References}

1. Heine, A.; Irmer, R. H.; Stalke, D., Structure and Crystal Packing of 4-Aminobenzonitriles and 4-Amino-3,5-Dimethylbenzonitriles at Various Temperatures. Acta. Cryst. 1994, B50, 363373.

2. Dekhtyar, M.; Rettig, W.; Rothe, A.; Kurdyukov, V.; Tolmachev, A., Variation of Donor and Acceptor Strength in Analogues of Brooker's Merocyanine and Generalization to Various Classes of Charge Transfer Compounds. J. Phys. Chem. A 2019, 123, 2694-2708.

3. Jayanty, S.; Radhakrishnan, T. P., Modeling Molecule-in-a-Crystal: The Case of Push-Pull Quinonoids. Chem. Mater. 2001, 13, 2460-2462. 